\title{
Bone Marrow Pathology Predicts Mortality in Chronic Hemodialysis Patients
}

\author{
Cheng-Hao Weng, ${ }^{1,2,3}$ Kuan-Ying Lu, ${ }^{1,2}$ Ching-Chih Hu, ${ }^{2,4}$ Wen-Hung Huang, ${ }^{1,2,3}$ \\ I-Kwan Wang, ${ }^{5,6}$ and Tzung-Hai Yen ${ }^{1,2,3,7}$ \\ ${ }^{1}$ Department of Nephrology and Division of Clinical Toxicology, Chang Gung Memorial Hospital, Linkou 333, Taiwan \\ ${ }^{2}$ College of Medicine, Chang Gung University, Taoyuan 333, Taiwan \\ ${ }^{3}$ Kidney Research Center, Chang Gung Memorial Hospital, Linkou 333, Taiwan \\ ${ }^{4}$ Department of Hepatogastroenterology and Liver Research Unit, Chang Gung Memorial Hospital, Keelung 204, Taiwan \\ ${ }^{5}$ Department of Nephrology, China Medical University Hospital, Taichung 404, Taiwan \\ ${ }^{6}$ College of Medicine, China Medical University, Taichung 404, Taiwan \\ ${ }^{7}$ Center for Tissue Engineering, Chang Gung Memorial Hospital, Linkou 333, Taiwan
}

Correspondence should be addressed to Tzung-Hai Yen; m19570@adm.cgmh.org.tw

Received 6 November 2014; Revised 10 January 2015; Accepted 9 February 2015

Academic Editor: Urszula Demkow

Copyright (c) 2015 Cheng-Hao Weng et al. This is an open access article distributed under the Creative Commons Attribution License, which permits unrestricted use, distribution, and reproduction in any medium, provided the original work is properly cited.

\begin{abstract}
Introduction. A bone marrow biopsy is a useful procedure for the diagnosis and staging of various hematologic and systemic diseases. The objective of this study was to investigate whether the findings of bone marrow studies can predict mortality in chronic hemodialysis patients. Methods. Seventy-eight end-stage renal disease patients on maintenance hemodialysis underwent bone marrow biopsies between 2000 and 2011, with the most common indication being unexplained anemia followed by unexplained leukocytosis and leukopenia. Results. The survivors had a higher incidence of abnormal megakaryocyte distribution $(P=0.001)$, band and segmented cells $(P=0.021)$, and lymphoid cells $(P=0.029)$ than the nonsurvivors. The overall mortality rate was $38.5 \%(30 / 78)$, and the most common cause of mortality was sepsis $(83.3 \%)$ followed by respiratory failure (10\%). In multivariate Cox regression analysis, both decreased (OR 3.714, 95\% CI 1.671-8.253, $P=0.001$ ) and absent (OR 9.751, 95\% CI 2.030-45.115, $P=0.004$ ) megakaryocyte distribution (normal megakaryocyte distribution as the reference group), as well as myeloid/erythroid ratio (OR 1.054, CI 1.012-1.098, $P=0.011$ ), were predictive of mortality. Conclusion. The results of a bone marrow biopsy can be used to assess the pathology, and, in addition, myeloid/erythroid ratio and abnormal megakaryocyte distribution can predict mortality in chronic hemodialysis patients.
\end{abstract}

\section{Introduction}

A bone marrow biopsy is a useful procedure for the diagnosis and staging of various hematologic diseases and in the assessment of bone marrow cellularity, cellular morphology, maturation, and the possibility of occult infection [1]. In ESRD patients, bone marrow studies are especially useful to evaluate the iron store in patients with anemia. Anemia in ESRD patients results from the reduced kidney production of erythropoietin (EPO) and changes in iron homeostasis which can lead to iron deficiency. Thus, routine monitoring of iron store is crucial for the adequate management of anemia in these patients [2]. Bone marrow fibrosis with a concomitant reduction in space for erythrogenesis induced by secondary hyperparathyroidism increases the dose of EPO required to maintain an adequate response [3]. The finding of bone marrow fibrosis in bone marrow studies in ESRD patients with anemia can allow for the administration of an adequate dosage of calcitriol or its analogs or parathyroidectomy. Except for those with anemia, other indications for bone marrow studies in ESRD patients are the same as in the general population. Therefore, the objective of this study was to investigate whether the findings of bone marrow studies can predict mortality in chronic hemodialysis patients. 


\section{Material and Methods}

2.1. Ethics Statement. This retrospective observational study complied with the guidelines of the Declaration of Helsinki and was approved by the Medical Ethics Committee of Chang Gung Memorial Hospital, a tertiary referral center located in northern Taiwan. Since this study involved a retrospective review of existing data, approval from the Institutional Review Board was obtained, but without specific informed consent from the patients. Furthermore, all data were securely protected (by delinking identifying information from the main data sets) and made available only to investigators, and they were also analyzed anonymously. The Institutional Review Board of Chang Gung Memorial Hospital specifically waived the need for consent for these studies. Finally, all primary data were collected according to procedures outlined in epidemiology guidelines that strengthen the reporting of observational studies. This policy was based on previous publications [4-6].

2.2. Patients. In total, 78 ESRD patients on maintenance hemodialysis underwent bone marrow biopsies between 2000 and 2011. Demographic, hematological, biochemical, and dialysis related data were obtained at the time of the bone marrow biopsies for cross-sectional analysis. Causes of death and mortality rates were also analyzed for each subgroup.

2.3. Inclusion and Exclusion Criteria. Patients were included in this study if they were more than 18 years of age and if they had undergone hemodialysis for at least 3 months. Patients with the final diagnosis of hematological diseases such as leukemia and multiple myeloma were excluded. The indications for the bone marrow biopsy are listed in Table 2 .

2.4. Bone Marrow Biopsy Specimen Preparation. The procedure was based on the guidelines published by the International Council for Standardization in Hematology [7]. Bone marrow biopsy specimens were processed with fixation and decalcification. After decalcification, the specimens were embedded in paraffin wax and sections were cut on a microtome. The biopsy sections were stained with hematoxylin and eosin.

2.5. Bone Marrow Biopsy Sections Microscopy Examination. The procedure was based on the guidelines published by the International Council for Standardization in Hematology [7]. Two to four sections were routinely reviewed. The percentage of cellularity was obtained by estimating the proportion of cells occupying the total marrow cavity. The sections were viewed initially at low power $(\times 40-\times 100)$ for adequacy, pattern, cellularity, presence of focal lesions, number of megakaryocytes, abnormal cell clusters and location, bone structure (trabecular number and thickness), and osteoclastic and osteoblastic activity. The sections were subsequently viewed under higher magnification $(\times 200-\times 400)$ to assess hematopoietic activity (e.g., erythroid, myeloid, megakaryocytic lineages, lymphoid cells, plasma cells, and macrophages) and cytological detail. Higher magnifications of $\times 600-\times 1000$ were used to assess fine cytological details such as intracellular granules and Auer rods.
2.6. Definition of Bone Marrow Cellularity. Bone marrow contains hematopoietic stem cells and stromal cells (mostly adipocytes) [8], and marrow cellularity is the volume ratio of hematopoiesis and fat. The normal cellularity of adult hematopoietic bone marrow ranges from 30 to $70 \%$, and this changes under pathological conditions. Hypercellular marrow is defined as more than $70 \%$, normocellular marrow as $30-70 \%$, and hypocellular marrow as under $30 \%$ bone marrow [9].

2.7. Definition of Normal, Increased, and Decreased Megakaryocyte Distribution. Normally, about 5 to 10 megakaryocytes are seen per microscopic field at low power magnification (10x objective). Clusters of megakaryocytes usually indicate megakaryocytic hyperplasia or increased megakaryocyte distribution. Less than 2 megakaryocytes per low power field means megakaryocytic hypoplasia [9] or decreased megakaryocyte distribution. An abnormal megakaryocyte distribution was defined as an increase, decrease, or absence of the distribution of megakaryocytes.

2.8. Definition of Mortality and Survival. The definition of mortality and survival in this study was mortality or survival after the bone marrow biopsy procedure.

2.9. Definition of Hemodialysis Adequacy $K t / V$. The $K t / V$ is used to quantify the adequacy of hemodialysis treatment, where $K$ represents the dialyzer clearance of urea, $t$ represents dialysis time, $V$ represents the volume of distribution of urea which is approximately equal to the patient's total volume of body water [10].

2.10. Statistical Analysis. Data were expressed as mean \pm standard deviation or number and percentage in parentheses unless otherwise stated. All variables were tested for normal distribution using the Kolmogorov-Smirnov test. The Student's $t$-test was used to compare the means of continuous variables and normally distributed data. Otherwise, the Mann-Whitney $U$ test was used for nonnormally distributed data. Categorical data were analyzed using the chi-square test. Finally, risk factors were assessed by univariate Cox regression analysis, and variables that were statistically significant $(P<0.05)$ were included in multivariate analysis by applying multiple Cox regression analysis based on forward elimination of data [11]. The cumulative survival curves as a function of time were generated using the Cox regression survival approach. All statistical tests were 2-tailed, with $P$ values less than 0.05 being considered statistically significant. Data were analyzed using SPSS 12.0 software for Windows (SPSS, Inc., Chicago, IL).

\section{Results}

3.1. Subject Characteristics. The overall mortality rate was $38.5 \%$ (30/78) (Table 1). The mean age of the ESRD patients who underwent a bone marrow biopsy was $63.5 \pm 17.2$ years, and the patients were followed up for $19.3 \pm 26.8$ months. There were no significant differences in baseline variables between the survivors and nonsurvivors. 
TABLE 1: Baseline data of the patients stratified according to survival status $(n=78)$.

\begin{tabular}{|c|c|c|c|c|}
\hline Variable & Total $(n=78)$ & Survivors $(n=48)$ & Nonsurvivors $(n=30)$ & $P$ value \\
\hline Age (years) & $63.5 \pm 17.2$ & $61.7 \pm 18.06$ & $66.4 \pm 15.6$ & 0.237 \\
\hline Female gender, $n(\%)$ & $36(46.2 \%)$ & $21(43.8 \%)$ & $27(56.3 \%)$ & 0.645 \\
\hline Hypertension, $n(\%)$ & $58(74.4 \%)$ & $38(79.2 \%)$ & $20(66.7 \%)$ & 0.288 \\
\hline Diabetes mellitus, $n(\%)$ & $30(38.5)$ & $16(33.3 \%)$ & $14(46.7 \%)$ & 0.339 \\
\hline Smoking habit, $n(\%)$ & $19(24.4 \%)$ & $12(25.0 \%)$ & $7(23.3 \%)$ & 1.000 \\
\hline Alcohol consumption, $n(\%)$ & $11(14.1 \%)$ & $5(10.4 \%)$ & $6(20.0 \%)$ & 0.319 \\
\hline Antihypertensive agents, $n(\%)$ & $58(74.4 \%)$ & $36(75.0 \%)$ & $22(73.3 \%)$ & 1.000 \\
\hline Lipid-lowering agents, $n(\%)$ & $4(5.1 \%)$ & $3(6.2 \%)$ & $1(3.3 \%)$ & 1.000 \\
\hline Iron, $n(\%)$ & $59(75.6 \%)$ & $38(79.2 \%)$ & $21(70.0 \%)$ & 0.421 \\
\hline Erythropoietin, $n(\%)$ & $48(61.5 \%)$ & $32(66.7 \%)$ & $16(53.3 \%)$ & 0.339 \\
\hline Duration of followup (months) & $19.3 \pm 26.8$ & $22.4 \pm 30.0$ & $14.3 \pm 22.4$ & 0.196 \\
\hline$K t / V$ & $2.1 \pm 0.8$ & $2.09 \pm 0.6$ & $2.21 \pm 0.4$ & 0.120 \\
\hline
\end{tabular}

TABLE 2: Indications for bone marrow biopsy stratified according to survival status $(n=78)$.

\begin{tabular}{lccc}
\hline Variable & Total $(n=78)$ & Survivors $(n=48)$ & Nonsurvivors $(n=30)$ \\
\hline Unexplained anemia, $n(\%)$ & $35(44.9 \%)$ & $23(47.9 \%)$ & $12(40.0 \%)$ \\
Unexplained leukocytosis and leukopenia, $n(\%)$ & $11(14.1 \%)$ & $5(10.4 \%)$ & $6(20.0 \%)$ \\
Fever of unknown origin, $n(\%)$ & $5(5.1 \%)$ & $2(4.2 \%)$ & $3(10.0 \%)$ \\
Suspected multiple myeloma, $n(\%)$ & $11(14.1 \%)$ & $8(16.7 \%)$ & $3(10.0 \%)$ \\
Suspected lymphoma, $n(\%)$ & $7(9.0 \%)$ & $3(6.3 \%)$ & $4(13.3 \%)$ \\
Pancytopenia, $n(\%)$ & $15(19.2 \%)$ & $10(20.8 \%)$ & $5(16.7 \%)$ \\
Thrombocytosis, $n(\%)$ & $1(1.3 \%)$ & $1(2.1 \%)$ & $0(0.0 \%)$ \\
Thrombocytopenia, $n(\%)$ & $7(17.9 \%)$ & $5(10.4 \%)$ & $2(6.7 \%)$ \\
Suspected hemolytic-uremic syndrome, $n(\%)$ & $1(1.3 \%)$ & $1(2.1 \%)$ & $0(0.0 \%)$ \\
\hline
\end{tabular}

${ }^{*} P<0.05$.

Unexplained anemia (44.9\%) was the most common indication for bone marrow biopsy in both the survivors $(47.9 \%)$ and nonsurvivors $(40.0 \%)$ (Table 2$)$. There were also no significant differences in the indications for a biopsy between the survivors and nonsurvivors. Furthermore, there were no significant differences in the laboratory variables between the survivors and nonsurvivors (Table 3 ).

3.2. Bone Marrow Biopsy Findings. The survivors had a higher incidence of abnormal megakaryocyte distribution $(P=$ $0.001)$, band and segmented cells $(P=0.021)$, and lymphoid cells $(P=0.029)$ compared to the nonsurvivors (Table 4$)$.

3.3. Causes of Mortality. The overall mortality rate was $38.5 \%$ $(30 / 78)$ and the most common cause of mortality was sepsis (83.3\%) followed by respiratory failure (10\%) (Table 5).

3.4. Predictors of Mortality. Univariate Cox regression analysis identified that systolic blood pressure, low bone marrow cellularity, megakaryocyte distribution, and $\mathrm{M} / \mathrm{E}$ ratio were significantly associated with mortality (Table 6). In a multivariate Cox regression model, decreased (OR 3.714, 95\% CI 1.671-8.253, $P=0.001$ ) and absent (OR 9.751, 95\%
CI 2.030-45.115, $P=0.004)$ megakaryocyte distribution (normal megakaryocyte distribution as the reference group), as well as M/E ratio (OR 1.054, CI 1.012-1.098, $P=0.011$ ), were predictive of mortality. The survival analysis showed a significantly higher cumulative rate of mortality in the patients with a decreased and absent megakaryocyte distribution compared with those with a normal megakaryocyte distribution (Figure 1).

\section{Discussion}

The $\mathrm{M} / \mathrm{E}$ ratio is relevant to bone marrow function and also to diseases of bone marrow and peripheral blood such as leukemia and anemia. A normal $\mathrm{M} / \mathrm{E}$ ratio is around $3: 1$, which may increase in patients with myelogenous leukemia and sepsis, decrease in patients with polycythemias, and reverse in cases of thalassemia [9]. In the current study, the $\mathrm{M} / \mathrm{E}$ ratio of the nonsurvivors was 5.12 , which is higher than that of the survivors (2.62; although not statistically significant) and higher than normal range. Furthermore, the most common cause of mortality in our patients was sepsis (83.3\%) and the increase in $\mathrm{M} / \mathrm{E}$ ratio may suggest that there was occult infection. Therefore, in a patient with high bone 
TABLE 3: Laboratory findings of the patients stratified according to survival status $(n=78)$.

\begin{tabular}{|c|c|c|c|c|}
\hline Variable & Total $(n=78)$ & Survivors $(n=48)$ & Nonsurvivors $(n=30)$ & $P$ value \\
\hline Red blood cell $\left(10^{6} / \mathrm{uL}\right)$ & $2.93 \pm 0.70$ & $2.90 \pm 0.70$ & $2.97 \pm 0.72$ & 0.663 \\
\hline Hemoglobin (g/dL) & $8.46 \pm 1.83$ & $8.41 \pm 1.94$ & $8.54 \pm 1.67$ & 0.757 \\
\hline Hematocrit (\%) & $25.53 \pm 5.50$ & $25.45 \pm 5.80$ & $25.66 \pm 5.10$ & 0.867 \\
\hline Mean corpuscular volume (fL) & $88.22 \pm 7.43$ & $88.56 \pm 6.85$ & $87.67 \pm 6.37$ & 0.609 \\
\hline Mean corpuscular hemoglobin (pg/cell) & $29.25 \pm 2.48$ & $29.24 \pm 2.35$ & $29.21 \pm 2.90$ & 0.954 \\
\hline Mean corpuscular hemoglobin concentration $(\mathrm{g} / \mathrm{dL})$ & $33.20 \pm 1.38$ & $33.09 \pm 1.25$ & $33.38 \pm 1.57$ & 0.375 \\
\hline Red blood cell distribution width (\%) & $16.09 \pm 3.03$ & $16.65 \pm 2.91$ & $15.26 \pm 3.06$ & 0.051 \\
\hline Platelet $\left(10^{3} / \mathrm{uL}\right)$ & $167.07 \pm 162.17$ & $191.09 \pm 182.44$ & $129.43 \pm 117.14$ & 0.104 \\
\hline White blood cells $\left(10^{3} / \mathrm{uL}\right)$ & $8.21 \pm 6.97$ & $7.76 \pm 5.75$ & $8.89 \pm 8.56$ & 0.494 \\
\hline Blast cells $(\%)$ & $2.00 \pm 0.00$ & $2.00 \pm 0.00$ & & \\
\hline Myelocytes (\%) & $1.46 \pm 1.11$ & $1.13 \pm 0.63$ & $1.64 \pm 1.31$ & 0.484 \\
\hline Metamyelocytes (\%) & $2.39 \pm 1.14$ & $2.50 \pm 0$ & $2.36 \pm 1.31$ & 0.888 \\
\hline Band cells (\%) & $2.58 \pm 2.32$ & $2.73 \pm 2.65$ & $2.50 \pm 2.23$ & 0.831 \\
\hline Segmented cells (\%) & $67.71 \pm 20.80$ & $68.77 \pm 19.16$ & $66.16 \pm 23.25$ & 0.600 \\
\hline Eosinophils (\%) & $3.88 \pm 5.00$ & $4.60 \pm 5.80$ & $2.33 \pm 1.86$ & 0.123 \\
\hline Basophils (\%) & $0.94 \pm 1.42$ & $1.02 \pm 1.62$ & $0.78 \pm 0.90$ & 0.589 \\
\hline Monocytes (\%) & $7.43 \pm 5.52$ & $8.19 \pm 5.77$ & $6.28 \pm 4.18$ & 0.130 \\
\hline Lymphocytes (\%) & $19.01 \pm 16.92$ & $17.71 \pm 15.06$ & $20.88 \pm 19.40$ & 0.435 \\
\hline Atypical lymphocytes (\%) & $1.83 \pm 1.34$ & $1.70 \pm 1.26$ & $1.93 \pm 1.48$ & 0.786 \\
\hline Alanine aminotransferase (U/L) & $19.16 \pm 19.35$ & $21.30 \pm 22.36$ & $15.94 \pm 10.78$ & 0.369 \\
\hline Blood urea nitrogen $(\mathrm{mg} / \mathrm{dL})$ & $77.95 \pm 51.71$ & $74.46 \pm 54.80$ & $83.30 \pm 47.05$ & 0.486 \\
\hline Creatinine $(\mathrm{mg} / \mathrm{dL})$ & $8.22 \pm 4.60$ & $8.66 \pm 4.87$ & $7.56 \pm 4.15$ & 0.320 \\
\hline Uric acid (mg/dL) & $7.67 \pm 3.93$ & $7.61 \pm 3.72$ & $7.84 \pm 4.92$ & 0.915 \\
\hline Calcium (mg/dL) & $8.80 \pm 1.30$ & $9.03 \pm 1.28$ & $8.45 \pm 1.28$ & 0.068 \\
\hline Phosphorus (mEq/L) & $5.33 \pm 2.56$ & $5.04 \pm 2.27$ & $5.74 \pm 2.91$ & 0.261 \\
\hline Potassium (mEq/L) & $4.34 \pm 0.93$ & $4.39 \pm 0.96$ & $4.26 \pm 0.90$ & 0.558 \\
\hline Bicarbonate (mEq/L) & $20.65 \pm 4.53$ & $21.84 \pm 4.32$ & $18.96 \pm 4.60$ & 0.207 \\
\hline Serum iron (ug/dL) & $35.20 \pm 22.88$ & $31.20 \pm 20.16$ & $36.13 \pm 34.15$ & 0.563 \\
\hline Total iron binding capacity (ug/dL) & $202.40 \pm 59.69$ & $185.30 \pm 47.53$ & $245.27 \pm 60.12$ & 0.227 \\
\hline Ferritin $(\mathrm{ng} / \mathrm{mL})$ & $837.90 \pm 367.00$ & $875.18 \pm 312.12$ & $749.85 \pm 412.12$ & 0.312 \\
\hline
\end{tabular}

marrow $\mathrm{M} / \mathrm{E}$ ratio, the possibility of occult infection should be taken into consideration.

The M/E ratio has also been reported to be higher in patients with erythroid hypoplasia, which can be caused by chronic inflammation [12], inadequate erythropoietin, or anti-erythropoietin antibodies in ESRD patients. Chan et al. [13] showed that growth of erythroid and granulocytic colonies was superior when cultured with nocturnal home hemodialysis (five to six times a week, 6 to 8 hours per session) plasma compared with conventional hemodialysis plasma and that intensification of the dosage of dialysis was associated with upregulation of the genes responsible for hematopoietic progenitor cell mobilization and growth and production of red blood cells. The conversion from conventional hemodialysis (three times a week, 4 hours per session) to nocturnal home hemodialysis has been reported to result in a three- to fourfold increase in urea clearance [14]. A direct relationship between the dosage of hemodialysis and the responsiveness of bone marrow in patients with ESRD has also been reported. Therefore, inadequate dialysis can be a cause of erythroid hypoplasia and result in anemia, which directly contributes to significant morbidity and mortality in ESRD patients [15].

The processes of megakaryocytopoiesis and platelet production occur within a complex bone marrow microenvironment where chemokines, cytokines, and adhesive interactions play a major role. Besides thrombopoietin, which is the main physiological regulator of megakaryocytopoiesis, other growth factors that stimulate megakaryocyte growth alone or in combination with EPO include granulocytemacrophage colony-stimulating factor, interleukin-3 (IL-3), IL-6, IL-11, stem cell factor, Fms-like tyrosine kinase 3 ligand, fibroblast growth factor, and EPO [16, 17]. On the other hand, transforming growth factor-betal, platelet factor 4, IL4, and Src kinase inhibitors have been shown to negatively regulate megakaryocyte proliferation by inducing megakaryocyte differentiation and functional platelet-like fragment formation in vitro $[18,19]$. Alvarez-Lara et al. showed that in uremic patients, only $5.1 \pm 2.1 \%$ of the $\mathrm{T}$ lymphocytes contained interferon gamma (Th1 cells), while $61.9 \pm 14.8 \%$ contained IL-4 (Th2 cells) $(P<0.0001)[20]$. More IL-4 may be produced in patients receiving inadequate dialysis. 
TABLE 4: Bone marrow biopsy findings stratified according to survival status $(n=78)$.

\begin{tabular}{|c|c|c|c|c|c|}
\hline Variable & \multicolumn{2}{|c|}{ Total $(n=78)$} & \multirow{2}{*}{$\begin{array}{c}\text { Survivors }(n=48) \\
20(41.7)\end{array}$} & \multirow{2}{*}{$\begin{array}{c}\text { Nonsurvivors }(n=30) \\
20(66.7)\end{array}$} & \multirow{2}{*}{$P$ value } \\
\hline \multirow{3}{*}{ Cellularity, $n(\%)$} & Hypocellularity & $40(51.3)$ & & & \\
\hline & Normocellularity & $23(29.5)$ & $18(37.5)$ & $5(16.7)$ & \multirow{2}{*}{0.077} \\
\hline & Hypercellularity & $15(19.2)$ & $10(20.8)$ & $5(16.7)$ & \\
\hline \multirow{4}{*}{ Megakaryocyte distribution, $n(\%)$} & Normal & $57(73.1)$ & $39(81.3)$ & $18(60.0)$ & \multirow{4}{*}{$0.001^{* *}$} \\
\hline & Increased & $6(7.7)$ & $6(6.3)$ & $0(0)$ & \\
\hline & Decreased & $13(16.7)$ & $3(6.3)$ & $10(33.3)$ & \\
\hline & Absence & $2(2.6)$ & $0(0)$ & $2(6.7)$ & \\
\hline \multirow{2}{*}{ Morphology, megakaryocytes, $n(\%)$} & Normal & $77(98.7)$ & $48(100.0)$ & $29(96.7)$ & \multirow{2}{*}{0.385} \\
\hline & Dysplasia & $1(1.3)$ & $0(0)$ & $1(3.3)$ & \\
\hline $\mathrm{M} / \mathrm{E}$ ratio & \multicolumn{2}{|c|}{$3.5 \pm 5.98$} & $2.62 \pm 1.64$ & $5.12 \pm 9.30$ & 0.154 \\
\hline Myeloid series (\%) & \multicolumn{2}{|c|}{$50.73 \pm 17.28$} & $53.06 \pm 16.21$ & $47.08 \pm 18.53$ & 0.140 \\
\hline Blast cells (\%) & \multicolumn{2}{|c|}{$0.90 \pm 1.06$} & $1.02 \pm 1.19$ & $0.70 \pm 0.77$ & 0.213 \\
\hline Promyelocytes (\%) & \multicolumn{2}{|c|}{$2.38 \pm 3.16$} & $2.47 \pm 3.71$ & $2.22 \pm 1.87$ & 0.743 \\
\hline Myelocytes + metamyelocytes (\%) & \multicolumn{2}{|c|}{$18.52 \pm 9.81$} & $18.08 \pm 9.29$ & $19.23 \pm 10.70$ & 0.618 \\
\hline Band cell + segmental cell (\%) & \multicolumn{2}{|c|}{$29.58 \pm 14.16$} & $32.48 \pm 14.38$ & $24.94 \pm 12.70$ & $0.021^{*}$ \\
\hline \multirow{2}{*}{ Morphology, myeloid series } & Normal & $77(98.7)$ & $48(100.0)$ & $29(96.7)$ & \multirow{2}{*}{0.194} \\
\hline & Abnormal & $1(1.3)$ & $0(0)$ & $1(3.3)$ & \\
\hline Erythroid series (\%) & \multicolumn{2}{|c|}{$23.99 \pm 12.49$} & $24.98 \pm 11.11$ & $22.39 \pm 14.48$ & 0.407 \\
\hline \multirow{2}{*}{ Morphology, erythroid series } & Normal & $77(98.7)$ & $48(100.0)$ & $29(96.7)$ & \multirow{2}{*}{0.328} \\
\hline & Dysplasia & $1(1.3)$ & $0(0)$ & $1(3.3)$ & \\
\hline Monohistiocytes (\%) & \multicolumn{2}{|c|}{$1.07 \pm 1.23$} & $1.10 \pm 1.32$ & $1.02 \pm 1.14$ & 0.814 \\
\hline Eosinophils (\%) & \multicolumn{2}{|c|}{$3.41 \pm 3.23$} & $3,91 \pm 3.19$ & $2.60 \pm 3.19$ & 0.085 \\
\hline Plasma cells (\%) & \multicolumn{2}{|c|}{$4.40 \pm 10.25$} & $2.64 \pm 2.24$ & $7.35 \pm 16.28$ & 0.139 \\
\hline Lymphoid cells (\%) & \multicolumn{2}{|c|}{$15.59 \pm 11.56$} & $12.99 \pm 7.77$ & $19.74 \pm 15.10$ & $0.029^{*}$ \\
\hline Iron store (grade) & \multicolumn{2}{|c|}{$2.41 \pm 1.33$} & $2.35 \pm 1.16$ & $2.54 \pm 1.66$ & 0.713 \\
\hline
\end{tabular}

${ }^{*} P<0.05,{ }^{* *} P<0.01$.

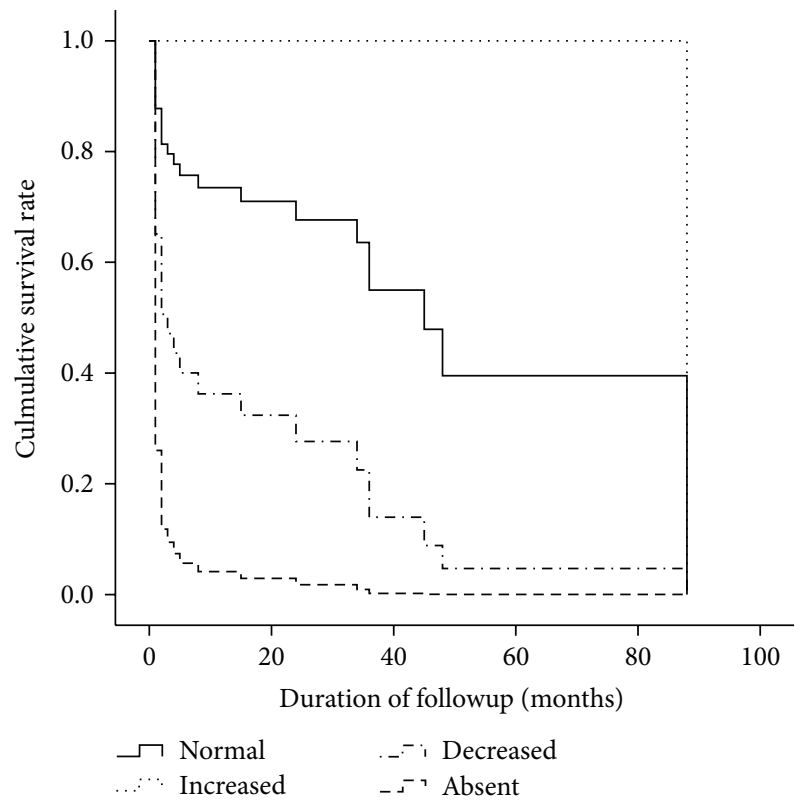

FIGURE 1: Kaplan-Meier survival probability estimates. The analysis showed a significantly higher cumulative mortality rate in the patients with a decreased and absent megakaryocyte distribution compared with those with a normal megakaryocyte distribution.
TABLE 5: Causes of mortality $(n=30)$.

\begin{tabular}{lc}
\hline Variable & \\
\hline Sepsis, $n(\%)$ & $25(83.3)$ \\
Respiratory failure, $n(\%)$ & $3(10.0)$ \\
Liver failure with encephalopathy, $n(\%)$ & $1(3.3)$ \\
Intracerebral hemorrhage & $1(3.3)$ \\
\hline
\end{tabular}

A decreased and absent megakaryocyte distribution were significant predictors of mortality in this study. There are many potential etiologies of decreased megakaryocytes such as cancer cells which infiltrate into the bone marrow and destroy megakaryocytes, aplastic anemia, toxic chemicals, radiation therapy or chemotherapy, genetic problems hindering the production of normal platelets, exposure to certain drugs or alcohol slowing the production of megakaryocytes, or simply viral infections. Nevertheless, the cause of a decreased or absent megakaryocytes in our dialysis patients remains unclear, and further research is warranted. Notably, Kantarjian et al. [21, 22] also showed that, in patients with chronic myelogenous leukemia, a decreased megakaryocyte distribution was a predictor of mortality and was associated with a poor prognosis. Therefore, the clinical significance of 
TABLE 6: Analysis of risk factors for mortality using Cox regression analysis $(n=78)$.

\begin{tabular}{|c|c|c|c|c|}
\hline & $B$ & SE & $\operatorname{Exp}(B)$ & $P$ value \\
\hline \multicolumn{5}{|l|}{ Univariate } \\
\hline Systolic blood pressure (mmHg) & -0.019 & 0.009 & $0.982(0.963-1.000)$ & $0.049^{*}$ \\
\hline Low bone marrow cellularity & -0.021 & 0.01 & $0.979(0.960-0.999)$ & $0.042^{*}$ \\
\hline \multicolumn{5}{|l|}{ Megakaryocyte distribution ${ }^{\#}$} \\
\hline Increased & -13.309 & 656.54 & 0.000 & 0.984 \\
\hline Decreased & 1.312 & 0.407 & $3.714(1.671-8.253)$ & $0.001^{* *}$ \\
\hline Absent & 2.259 & 0.791 & $9.571(2.030-45.115)$ & $0.004^{* *}$ \\
\hline $\mathrm{M} / \mathrm{E}$ ratio & 0.047 & 0.018 & $1.048(1.012-1.087)$ & $0.009^{* *}$ \\
\hline Plasma cell number & 0.026 & 0.011 & $1.027(1.005-1.049)$ & $0.014^{*}$ \\
\hline Lymphoid cell number & 0.036 & 0.013 & $1.037(1.010-1.064)$ & $0.006^{* *}$ \\
\hline \multicolumn{5}{|l|}{ Multivariate } \\
\hline \multicolumn{5}{|l|}{ Megakaryocyte distribution ${ }^{\#}$} \\
\hline Increased & -13.419 & 636.058 & 0.000 & 0.983 \\
\hline Decreased & 1.192 & 0.415 & $3.292(1.461-7.420)$ & $0.004^{* *}$ \\
\hline Absent & 2.335 & 0.808 & $10.333(2.119-50.376)$ & $0.004^{*}$ \\
\hline $\mathrm{M} / \mathrm{E}$ ratio & 0.053 & 0.021 & $1.054(1.012-1.098)$ & $0.011^{*}$ \\
\hline
\end{tabular}

megakaryocyte findings in bone marrow warrants further research.

\section{Conclusion}

The results of a bone marrow biopsy can be used to assess the pathology, and, in addition, $\mathrm{M} / \mathrm{E}$ ratio and abnormal megakaryocyte distribution can predict mortality in chronic hemodialysis patients.

\section{Conflict of Interests}

The authors declare that there is no conflict of interests regarding the publication of this paper.

\section{Authors' Contribution}

Cheng-Hao Weng and Kuan-Ying Lu contributed equally to this work.

\section{Acknowledgment}

Dr. Tzung-Hai Yen was funded by research Grants from Chang Gung Memorial Hospital (CMRPG3D0681, CMRPG3D0071-2, CMRPG3D0011, and CMRPG3E0361).

\section{References}

[1] B. J. Bain, "Bone marrow aspiration," Journal of Clinical Pathology, vol. 54, no. 9, pp. 657-663, 2001.

[2] L. A. Rocha, D. V. Barreto, F. C. Barreto et al., "Serum ferritin level remains a reliable marker of bone marrow iron stores evaluated by histomorphometry in hemodialysis patients," Clinical Journal of the American Society of Nephrology, vol. 4, no. 1, pp. 105-109, 2009.
[3] D. Brancaccio, M. Cozzolino, and M. Gallieni, "Hyperparathyroidism and anemia in uremic subjects: a combined therapeutic approach," Journal of the American Society of Nephrology, vol. 15, supplement 1, pp. S21-S24, 2004.

[4] C. Lin, T.-H. Yen, Y.-Y. Juang, J.-L. Lin, and S.-H. Lee, "Psychiatric comorbidity and its impact on mortality in patients who attempted suicide by paraquat poisoning during 2000-2010," PLoS ONE, vol. 9, no. 11, Article ID el12160, 2014.

[5] S. H. Liu, J. L. Lin, H. L. Shen et al., "Acute large-dose exposure to organophosphates in patients with and without diabetes mellitus: analysis of mortality rate and new-onset diabetes mellitus," Enviromental Health, vol. 13, no. 1, article 11, 2014.

[6] Y.-C. Hou, J.-L. Lin, W.-H. Huang et al., "Outcomes of patients with acetaminophen-associated toxic hepatitis at a far east poison center," SpringerPlus, vol. 2, no. 1, article 674, 2013.

[7] S.-H. Lee, W. N. Erber, A. Porwit, M. Tomonaga, and L. C. Peterson, "ICSH guidelines for the standardization of bone marrow specimens and reports," International Journal of Laboratory Hematology, vol. 30, no. 5, pp. 349-364, 2008.

[8] T.-H. Yen, M. R. Alison, R. A. Goodlad et al., "Epidermal growth factor attenuates tubular necrosis following mercuric chloride damage by regeneration of indigenous, not bone marrowderived cells," Journal of Cellular and Molecular Medicine, vol. 19, no. 2, pp. 463-473, 2015.

[9] G. S. Travlos, "Histopathology of bone marrow," Toxicologic Pathology, vol. 34, no. 5, pp. 566-598, 2006.

[10] F. A. Gotch and J. A. Sargent, "A mechanistic analysis of the National Cooperative Dialysis Study (NCDS)," Kidney International, vol. 28, no. 3, pp. 526-534, 1985.

[11] W.-H. Huang, J.-L. Lin, D.-T. Lin-Tan, C.-W. Hsu, K.-H. Chen, and T.-H. Yen, "Environmental lead exposure accelerates progressive diabetic nephropathy in type II diabetic patients," BioMed Research International, vol. 2013, Article ID 742545, 9 pages, 2013.

[12] I. C. Macdougall and A. C. Cooper, "Hyporesponsiveness to erythropoietic therapy due to chronic inflammation," European Journal of Clinical Investigation, Supplement, vol. 35, supplement 3, pp. 32-35, 2005. 
[13] C. T. Chan, P. P. Liu, S. Arab, N. Jamal, and H. A. Messner, "Nocturnal hemodialysis improves erythropoietin responsiveness and growth of hematopoietic stem cells," Journal of the American Society of Nephrology, vol. 20, no. 3, pp. 665-671, 2009.

[14] A. Pierratos, "New approaches to hemodialysis," Annual Review of Medicine, vol. 55, pp. 179-189, 2004.

[15] S. Khan and B. J. Pereira, "Hematocrit level associated mortality in hemodialysis patients, by Ma JZ, Ebben J, Xia H, Collins AJ. J Am Soc Nephrol 10:610-619, 1999," Seminars in Dialysis, vol. 13, no. 2, pp. 112-113, 2000.

[16] S. Bruno, M. Gunetti, L. Gammaitoni et al., "In vitro and in vivo megakaryocyte differentiation of fresh and ex-vivo expanded cord blood cells: rapid and transient megakaryocyte reconstitution," Haematologica, vol. 88, no. 4, pp. 379-387, 2003.

[17] K. Kaushansky, "Thrombopoietin: the primary regulator of platelet production," Blood, vol. 86, no. 2, pp. 419-431, 1995.

[18] B. J. Lannutti, N. Blake, M. J. Gandhi, J. A. Reems, and J. G. Drachman, "Induction of polyploidization in leukemic cell lines and primary bone marrow by Src kinase inhibitor SU6656," Blood, vol. 105, no. 10, pp. 3875-3878, 2005.

[19] G. Zauli and L. Catani, "Human megakaryocyte biology and pathophysiology," Critical Reviews in Oncology/Hematology, vol. 21, no. 1-3, pp. 135-157, 1995.

[20] M. A. Alvarez-Lara, J. Carracedo, R. Ramírez et al., “The imbalance in the ratio of Th1 and Th2 helper lymphocytes in uraemia is mediated by an increased apoptosis of Th1 subset," Nephrology Dialysis Transplantation, vol. 19, no. 12, pp. 3084-3090, 2004.

[21] H. M. Kantarjian, M. J. Keating, K. B. McCredie et al., "Old age: a sign of poor prognosis in patients with chronic myelogenous leukemia," Southern Medical Journal, vol. 80, no. 10, pp. 12281232, 1987.

[22] H. M. Kantarjian, T. L. Smith, K. B. McCredie et al., "Chronic myelogenous leukemia: a multivariate analysis of the associations of patient characteristics and therapy with survival," Blood, vol. 66, no. 6, pp. 1326-1335, 1985. 


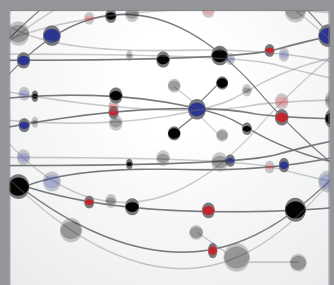

The Scientific World Journal
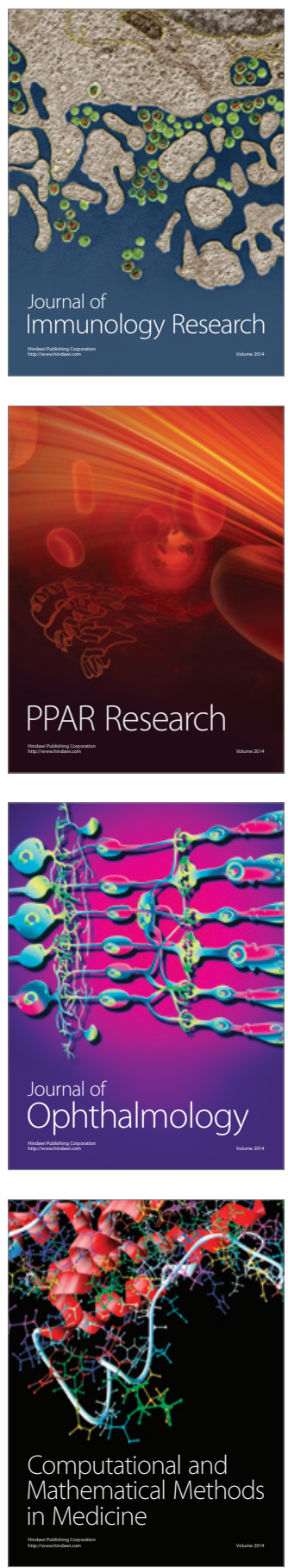

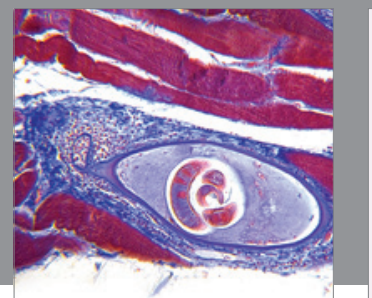

Gastroenterology

Research and Practice
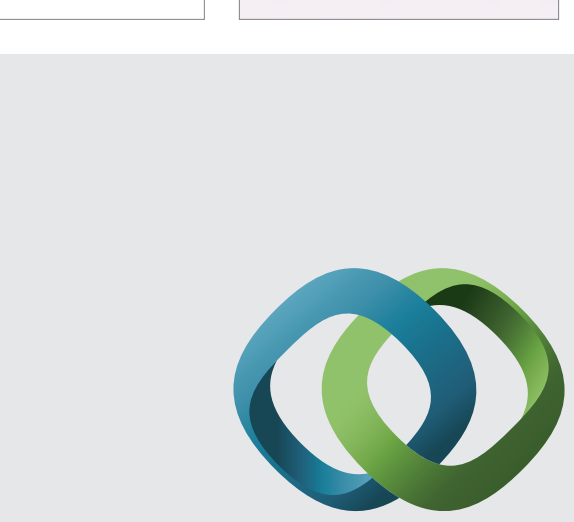

\section{Hindawi}

Submit your manuscripts at

http://www.hindawi.com
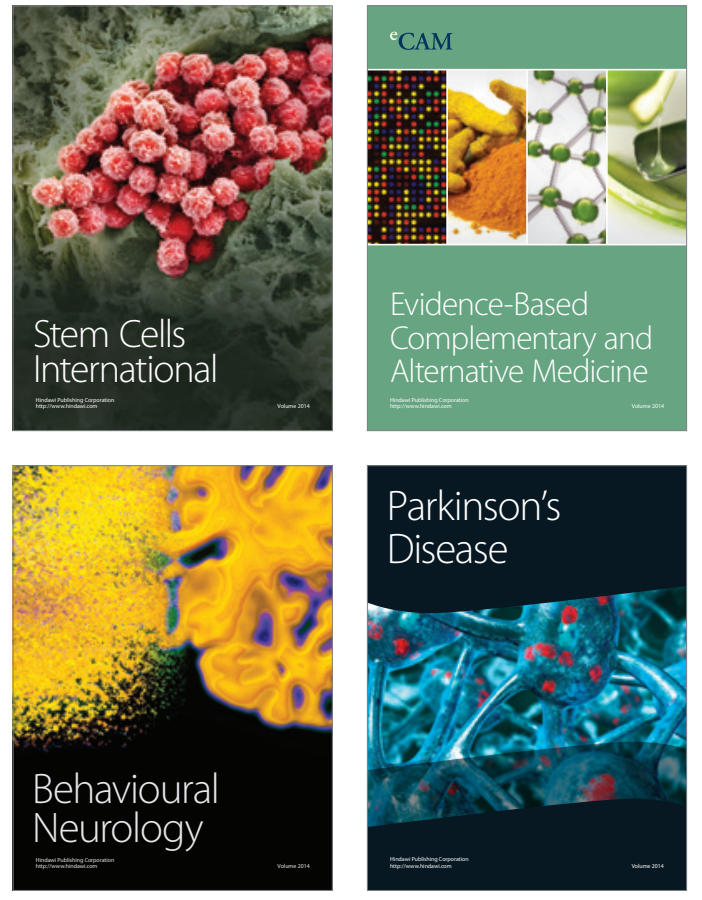
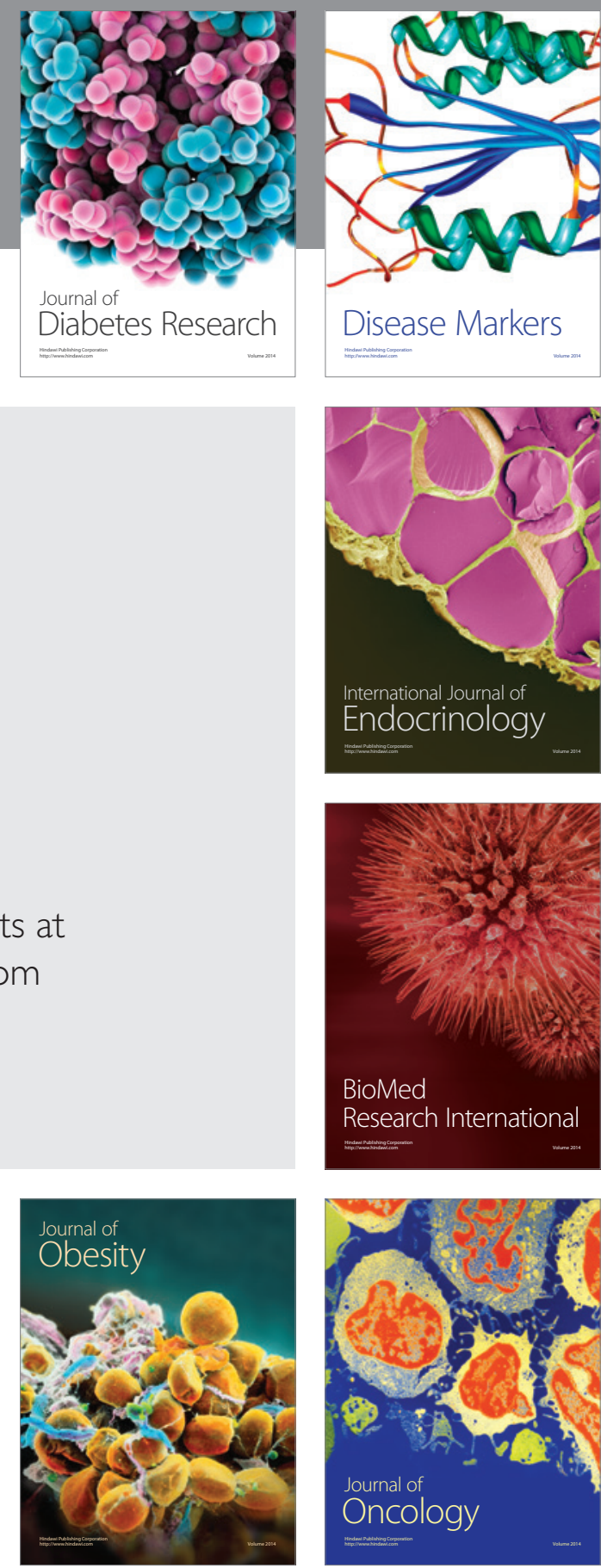

Disease Markers
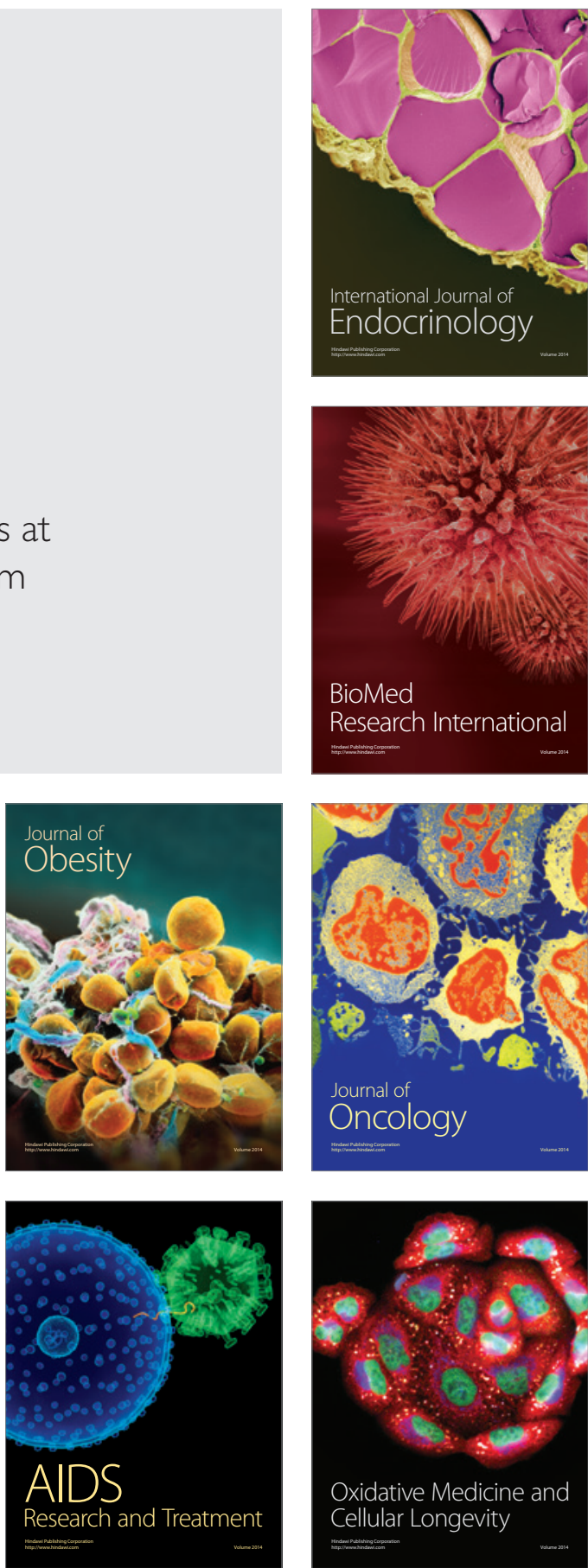